J. Clin. Chem. Clin. Biochem.

Vol. 19, 1981, pp. 375-378

\title{
Quantitative Bestimmung von Kreatinin im Harn mit Hochdruckflüssigkeitschromatographie
}

\author{
Von $A$. Hausen, D. Fuchs und $H$. Wachter \\ Institut für Medizinische Chemie und Biochemie der Universität Innsbruck
}

(Eingegangen am 24. Oktober 1980/16. Januar 1981)

Zusammenfassung: Eine einfache und spezifische Methode zur Kreatininbestimmung mit HPLC wird beschrieben. Die Trennung erfolgt an einer $\mathrm{C}_{18}$-reversed-phase-Säule. Die Detektion wird mit einem UV-Absorptionsspektralphotometer bei $235 \mathrm{~nm}$ vorgenommen. Die erhaltenen Zuverlässigkeitskriterien entsprechen bezüglich der Präzision und Richtigkeit den Richtlinien der Bundesärztekammer zur statistischen Qualitätskontrolle. Vergleichende Untersuchungen mit der Methode von Lim et al. ((1978), J. Chromatogr. 67, 182-187) ergaben einen Korrelationskoeffizienten von 0,994. Die Spezifität der Methode wurde zusätzlich durch das UV-Absorptionsspektrum der Kreatininfraktion bestätigt. Weiters wurden 28 Harnbestandteile auf ihre Interferenz mit Kreatinin untersucht.

\section{Determination of creatinine in urine by high performance liquid chromatography (HPLC)}

Summary: A simple, specific method is described for the determination of creatinine in urine by HPLC. A C $18^{-}$ reversed-phase column is used for the separation. Detection is with a spectral UV-absorption photometer at $235 \mathrm{~nm}$.

The results for the precision and the accuracy meet the guidelines of the statistical quality control. Comparative investigations using the method by Lim et al. ((1978), J. Chromatogr. 67, 182-187) matched well with a coefficient of reference of 0.994 . The specificity of the method was confirmed by the UV-absorption spectrum of creatinine isolated by HPLC. In addition, 28 urinary constituents were tested for possible interference.

\section{Einführung}

Kreatininbestimmungen in Harn und Serum besitzen große klinische Bedeutung. Żusätzlich werden vielfach andere Harnausscheidungsprodukte auf Kreatinin bezogen. Die im klinischen Routinebetrieb immer noch weitgehend eingesetzten Farbreaktionen, wie die JafféReaktion mit alkalischer Pikrinsäure (1) oder die Reakțion mit 3,5-Dinitrobenżoesäure sind wẹig spezifisch, dà sie von einigen endogenen Störfaktoren in Serum und Harn beeinflußt werden. Enzymatische Methoden $(2,3)$ konnten sich infolge geringer Haltbarkeit der Reagenzien und des relativ hohen Zeitaufwandes sowie der hohen Kosten noch nicht allgemein durchsetzen. Die HPĹC-Bestimmung des Kreatinins nach Vortrennung durch eine Kationenaustauschersäule an einer reversed-phase-Säule (4) und die Messung nạch Extraktion mit Acetonitril an einer Kationenaustauschersäule (5) eignen sich infolge guter Genauigkeit und Spezifität wohl als Vergleichsmethoden, sind jedoch aufgrund ihrer hohen Arbeitsintensität und ihres Zeitaufwandes als Routinemethoden wenig praktikabel.
Bei der quantitativen Messung von Neopterin als Tumormarker (6) erschien es zweckmäßig, Kreatinin als Bezugssubstanz in gleicher Weise mit HPLC zu bestimmen. Das ausgearbeitete Verfahren eignet sich ebenfalls sehr gut zur simultanen Bestimmung von Kreatinin und Harnsäure im Harn. Diese Methode zeichnet sich gegenüber anderen HPLC-Bestimmungen des Kreatinins $(4,5,7,8)$ dadurch aus, daß man zur Vortrennung käufliche Einmalsäulen verwendet. Im folgenden soll diese spezifische und genaue HPLC-Methode vorgestellt werden, die es ermöglicht, das sogenannte „wahre" Kreatinin rasch und einfach zu bestimmen.

\section{Material und Methoden}

Kollektivauswahl

Kreatinin wurde in einem Kollektiv von 15 gesunden Personen (7 Frauen, 8 Männer) sowohl enzymatisch als auch nach der von uns vorgeschlagenen HPLC-Methode bestimmt. Zusätzlich wurden zum Vergleich 46 Harne (26 Frauen, 20 Männer) mit der Methode von Lim et al. (4) und mit unserem Verfahren analysiert. 
Vorsäulen, Reagenzien und Lösungen

Vorsäule: Fertigsäule SEP-PAK- $\mathrm{C}_{18}, \mathrm{Fa}$. Waters, D-6440 Königstein. Diese Fertigsäulen sind nach Reinigung mit $2 \mathrm{ml}$ Methanol und anschließend mit $6 \mathrm{ml}$ Wasser insgesamt dreimal verwendbar.

\section{Geräte zur HPLC-Analyse}

Pumpe: Modell 110A, Fa. Altex, Berkeley, USA.

UV-Absorptionsdetektor: Spektralphotometer PM 2 DLC,

Fa. Zeiss, D-7082 Oberkochen.

Data-Processor: Chromatopac C-R1A, Shimadzu corporation, Kyoto 604, Japan.

\section{UV-Spektren}

Spektralphotometer Modell 25, Fa. Beckman, D-8000 München.

\section{Arbeitsvorschrift}

Etwa $10 \mathrm{ml}$ eines $24 \mathrm{~h}$-Urins oder einer beliebigen Urinprobe werden filtriert, $1,0 \mathrm{ml}$ des Filtrates werden auf $10 \mathrm{ml}$ aufgefüllt und $1,0 \mathrm{ml}$ des verdünnten Filtrates mit einer $10 \mathrm{ml}$ Glasspritze mit Luerlok-Anschluß durch eine käufliche SEP-PAK$\mathrm{C}_{18}$-Kartusche ( $\mathrm{Fa}$. Waters) gedrückt. Anschließend wird mit $4 \mathrm{ml}$ Wasser eluiert.

Das Gesamteluat ( $5 \mathrm{ml}$ ) wird gut gemischt und davon werden $20 \mu l$ in den Hochdruckflüssigkeitschromatographen injiziert.

Hoch druckflüssigkeitschroma tographie

Trennsäule: reversed-phase-Säule $\left(10 \mu\right.$-Bondapak $\mathrm{C}_{18}, \mathrm{Fa}$. Waters, $30 \mathrm{~cm} \times 3,9 \mathrm{~mm}$ i.D.), Temperatur $25^{\circ} \mathrm{C}$. Als mobile Phase dient wäßriger Puffer (pH 7,0;0,030 mol/1 Ammoniumacetat; $1,560 \mathrm{~mol} / 1 \mathrm{Methanol}$ ) bei einer Durchflußgeschwindigkeit von $2,0 \mathrm{ml} / \mathrm{min}$. Der UV-Detektor ist auf $235 \mathrm{~nm}$ eingestellt.

Die Flächenmessung erfolgt mit dem Integrator Chromatopac C-R1A. Ein typisches Chromatogramm einer gesunden Person ist in Abbildung 1 dargestellt.

\section{Kalibrierung}

Zur Kalibrierung wurde der Harn einer gesunden Person 1:10 verdünnt und mit Kreatininstammlösung auf Kreatininkonzentrationen von 0,88 bis $5,85 \mathrm{mmol} / \mathrm{l}$ aufgestockt. Jede dieser Standardlösungen wurde entsprechend der Arbeitsanleitung analysiert.
Im Standardkurvendiagramm (Abb. 2) zeichnet man die Peakfläche als Ordinate, den Kreatininzusatz in mmol/1 Harn als Abszisse ein. Die Standardkurve verhält sich im untersuchten Bereich mit einem Korrelationskoeffizienten von $r=0,996$ linear. Durch die Parallelverschiebung der Standardkurve läßt sich der Kreatiningehalt des zum Aufstocken verwendeten Harns eliminieren.

\section{Ergebnisse}

\section{Spezifität}

Die Spezifität der Bestimmung kann nur von stark polaren Substanzen beeinflußt werden, die sich bei der reversed-phase Trennung ähnlich wie.Kreatinin verhalten und gleichzeitig bei $235 \mathrm{~nm}$ absorbieren. In Tabelle 1 sind die auf Kreatinin bezogenen Retentionszeiten von 28 in Frage kommenden Substanzen angeführt. Diese überprüften Substanzen interferieren wegen ihren unterschiedlichen Retentionszeiten bzw. wegen ihrer bei $235 \mathrm{~nm}$ geringen UV-Absorption nicht.

Zum weiteren Nachweis der Reinheit der Kreatininfraktion wurde die kreatininhaltige Fraktion nach der HPLC-Trennung sowohl von Standard- als auch von Hamproben aufgefangen. Wir nahmen von beiden Fraktionen die UV-Absorptionsspektren auf. Die für beide Proben praktisch identischen UV-Absorptionsspektren mit genau übereinstimmenden Maxima bei $212 \mathrm{~nm}$ und $235 \mathrm{~nm}$ zeigen die Reinheit der Fraktion und dadurch ebenfalls die Spezifität der neuen HPLCBestimmung des Kreatinins. Die Spezifität der Methode

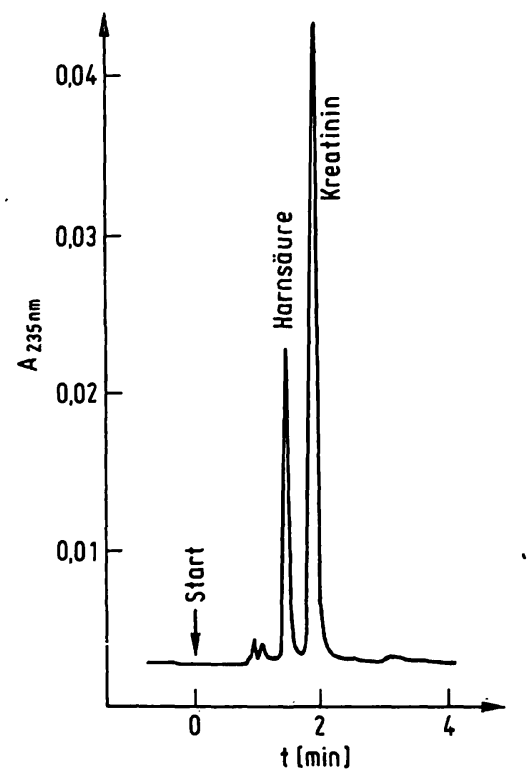

Abb. 1. Reversed-phase Chromatogramm eines Harnes. Trennsäule: reversed-phase $10 \mu$-Bondapak $\mathrm{C}_{18}$, Länge: $30 \mathrm{~cm}$; i. D.: $3,9 \mathrm{~mm}$. Temperatur: $25^{\circ} \mathrm{C}$. Mobile Phase: $0,030 \mathrm{~mol} / 1$ Ammoniumacetat mit 1,560 $\mathrm{mol} / 1 \mathrm{Methanol}$. Fluß: $2 \mathrm{ml} / \mathrm{min}$.

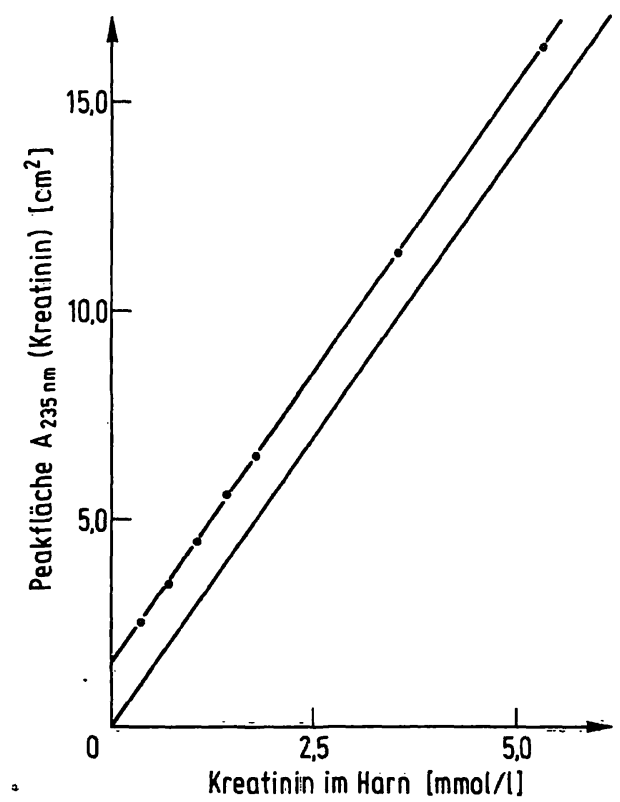

Abb. 2. Standardkurven zur hochdruckflüssigkeitschromatographischen Kreatininbestimmung im Harn.

Additionsstandardkuve ( $\cdot \cdots \cdot \cdot$ ) (Ham mit definierten Kreatininzusätzen) und

Standardkurve nach Parallelverschiebung durch den Nullpunkt (-). 
Tab. 1. Zusammenstellung von $28 \mathrm{im}$ Harn vorkommenden UVabsorbierenden polaren Substanzen und ihre auf Kreatinin relativierten Retentionszeiten.

\begin{tabular}{ll}
\hline Substanz & $\begin{array}{l}\text { Auf Kreatinin } \\
\text { relativierte } \\
\text { Retentionszeit }\end{array}$ \\
\hline Äpfelsäure & 0,46 \\
Oxalsäure & 0,48 \\
Citronensäure & 0,60 \\
Ascorbinsäure & 0,67 \\
Orotsäure & 0,74 \\
Allantoin & 0,75 \\
3-Hydroxyanthranilsäure & 0,78 \\
Harnsäure & 0,83 \\
Cytosin & 0,91 \\
Coffein & 0,91 \\
Uracil & 0,96 \\
Kreatinin & 1,00 \\
4-Aminohippursäure & 1,10 \\
Ribosyluracil & 1,15 \\
Glucose & 1,19 \\
Tyrosin & 1,21 \\
2,8-Dihydroxyadenin & 1,28 \\
Hypoxanthin & 1,29 \\
Isoguanin & 1,32 \\
Guanin & 1,34 \\
Xanthin & 1,34 \\
Kynurenin & 1,36 \\
Hippursäure & 1,41 \\
Thymin & 1,45 \\
5-Hydroxyindolessigsäure & 1,60 \\
7-Methylguanin & 2,38 \\
Adenin & 2,43 \\
Nicotinamid & 2,73 \\
Indoxylsulfat & 3,68 \\
\hline & \\
\hline & \\
\hline &
\end{tabular}

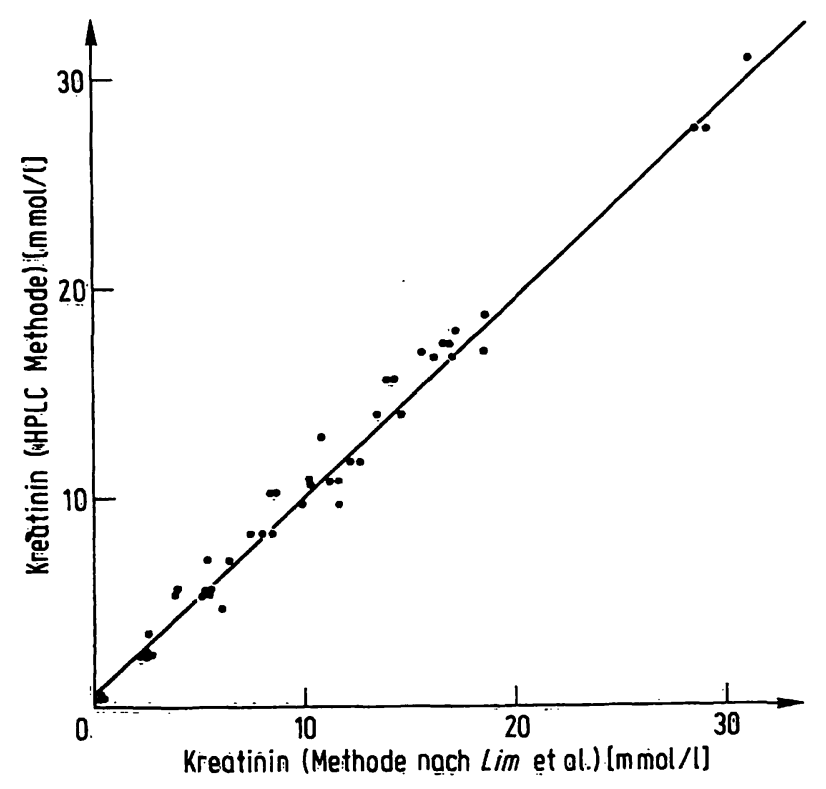

Abb. 3. Kotrelationsdiagramm der hochdruckflüssigkeitschromatographischen Kreatininbestimmung an reversedphase ( $y$-Werte) und den Werten der in 1.c. (4) beschriebenen Methode (x-Werte) im Harn. $n=46$.

$y=0,0594+0,981 x$

$r=0,994(p<0 ; 0005)$. wurde zusätzlich durch Vergleich mit der als spezifisch anerkannten $(9,10)$ Methode von Lim et al. (4) überprüft. Bei der Analyse von 46 Harnen mit beiden Methoden ergab sich ein Korrelationskoeffizient von $r=0,994$ (Abb. 3).

Ein Vergleich der neuen HPLC-Methode mit einer enzymatischen $(2,3)$ an 15 Harnen ergab mit einem Korrelationskoeffizienten von $r=0,985(p<0,0005)$ keinen Unterschied zwischen beiden Bestimmungen.

\section{Präzision}

Die Präzision in der Serie wurde durch 15-malige Analyse von zwei Harnen mit einem mittleren Gehalt von 8,04 mmol/l bzw. $18,12 \mathrm{mmol} / \mathrm{l} \mathrm{Kreatinin} \mathrm{bestimmt.} \mathrm{Es} \mathrm{er-}$ rechnete sich ein Variationskoeffizient in der Serie von 1,57\% bzw. 1,15\%. Die Präzision von Tag zu Tag wurde an drei Harnen unterschiedlicher Konzentration mit folgenden Variationskoeffizienten bestimmt:

$$
\begin{aligned}
& \text { VK }=2,59 \%(\bar{x}=18,74 \mathrm{mmol} / 1 ; n=12) \\
& \text { VK }=3,28 \%(\bar{x}=11,58 \mathrm{mmol} / 1 ; n=12) \\
& \text { VK }=3,70 \%(\bar{x}=9,99 \mathrm{mmol} / 1 ; n=12)
\end{aligned}
$$

\section{Richtigkeit}

Die Uberprüfung der Richtigkeit des Analysenverfahrens erfolgte durch Wiederauffindungsversuche. Hierzu versetzten wir Harn mit einer mittleren Kreatininkonzentration von $5,30 \mathrm{mmol} / \mathrm{l}$ mit zusätzlich $7,69 \mathrm{mmol} / 1$ Kreatinin. Wir fanden in 1:10 verdünntem Harn eine durchschnittliche Konzentration von $1,33 \mathrm{mmol} / 1$ Kreatinin. Dies entspricht einer Wiederauffindung von $102,39 \%$.

\section{Nachweisgrenzen}

In wäßrigen Kreatininlösungen konnten noch $1,8 \mu \mathrm{mol} / 1$. Kreatinin bei einem Peak/Rausch Verhältnis von 5:1 nachgewiesen werden. Da $20 \mu$ lösung aufgegeben wurden, entspricht dies $36 \mathrm{pmol}$ pro Injektion.

\section{Obere Grenze des Meßbereichs}

Bei Harnanalysen nach der Arbeitsvorschrift können Proben bis zu einer Konzentration von $36 \mathrm{mmol} / \mathrm{l}$ untersucht werden. Harne höherer Konzentration müssen weiter verdünnt werden, um bei der Absorptionsmessung im linearen Bereich zu bleiben.

\section{Praktikabilität}

Die Probenvorbereitung erfordert für 12 Analysen eine halbe Stunde. Alle 7 Minuten kann eine Harnprobe injiziert werden. Verunreinigungen mit Retentionszeiten über 7 Minuten, die eine Auswertung der folgenden 
Chromatogramme beeinträchtigen würden, sind aufgrund der Probenvorbehandlung nicht zu erwarten und wurden auch nicht beobachtet. Die Materialkosten sind minimal, was einen wesentlichen Vorteil gegenüber den enzymatischen Methoden darstellt.

\section{Diskussion}

Verglichen mit bisher publizierten HPLC-Bestimmungen des Kreatinins ist die von uns vorgeschlagene Methode einfacher, rascher und durch den Einsatz kommerzieller Fertigsäulen zeitsparender, wie es besonders für Routineuntersuchungen in Kliniken wünschenswert ist. Harnbestandteile mit längeren Retentionszeiten als Xanthin gelangen infolge der Probenvorbehandlung nicht auf die HPLC-Säule. Die nächste Probe kann also nach 7 Minuten injiziert werden, da keine Memoryeffekte auftreten. Das Verfahren ist auch weniger arbeits- und kostenintensiv als die enzymatischen Methoden, aber ebenso spezifisch wie diese.

$\mathrm{Da}$ die zu fordernde Spezifität nicht bei allen bisher üblichen Kreatininbestimmungen gewährleistet ist, wurde sie streng getestet. Durch Uberprïfung anderer Harnsubstanzen, durch Aufnahme von UV-Absorptionsspektren der Kreatininfraktion nach HPLC. Trennung von Standard- und Harnproben und durch Vergleich mit spezifischen Referenzmethoden konnte die Spezifität der von uns vorgeschlagenen Methode gezeigt werden.

Das hier beschriebene HPLC-Verfahren entspricht bezüglich der Spezifität, Präzision und Richtigkeit den Richtlinien der Bundesärztekammer zur statistischen Qualitätskontrolle (11). Hinzuweisen ist, daß mit diesem HPLC-Verfahren neben Kreatinin noch Harnsäure und einige weitere im Harn vorkommende Purine simultan bestimmt werden können (Hausen \& Wachter, in Vorbereitung).

\section{Literatur}

1. Chiou, W. L., Peng, G. W. \& Gadalla, M. A. F. (1978), J. Pharm. Sci. 67, 292-293.

2. Wahlefeld, A. W., Herz, G. \& Bergmeyer, H. U. (1972), Scand. J. Clin. Lab. Invest. 29, Suppl. 126, 30.1.

3. Wahlefeld, A. W., Holz, G. \& Bergmeyer, H. U. (1974), Methoden der enzymatischen Analyse, 3. Auflage Band II, pp. 1834-1838 (Bergmeyer, H. U. ed.), Verlag Chemie, Weinheim/Bergstr.

4. Lim, C. K., Richmond, W., Robinson, D. P. \& Brown, S. S. (1978), J. Chromatogr. 145, 41-49.

5. Chiou, W. L., Gadalla, M. A. F. \& Peng, G. W. (1978), J. Pharm. Sci. 67, 182-187.

6. Wachter, H., Hausen, A. \& Graßmayr, K, (1979), HoppeSeyler's Z. Physiol. Chem. 360, 1957-1960.
7. Soldin, J. S. \& Hill, J. G. (1978), Clin. Chem. 24, 747-750.

8. Brown, N. D., Sing, H. C., Neeley, W. E. \& Koetitz, S. E. (1977), Clin. Chem. 23, 1281-1283.

9. Buchanan, D. N., Sandra, K. T. \& Domino, E. F. (1979), J. Chromatogr. 163, 212-216.

10. Krstulovic, A. M., Bertani-Dziedzic, L. \& Caporusso, J. M. (1979), Clin. Chim. Acta 99, 189-194.

11. Richtlinien der Bundesärztekammer zur Durchführung von Maßnahmen der statistischen Qualitätskontrolle und von Ringversuchen im Bereich der Heilkunde (1970), Dt. Ärzteblatt 67, 2228-2231.

Prof. Dr. Helmut Wachter Institut für Medizinische Chemie und Biochemie der Universität Innsbruck Fritz-Pregl-Straße 3 A-6020 Innsbruck 